\title{
LHCf experiment: forward physics at LHC for cosmic rays study
}

\author{
M. Del Prete ${ }^{1,2, a}$, O. Adriani ${ }^{1,2}$, E. Berti ${ }^{1,2}$, L. Bonechi ${ }^{1,2}$, M. Bongi ${ }^{1,2}$, G. Castellini ${ }^{3}$, R.
} $D^{\prime}$ Alessandro ${ }^{1,2}$, M. Haguenauer ${ }^{4}$, Y. Itow ${ }^{5,6}$, K. Kasahara ${ }^{7}$, K. Kawade ${ }^{5}$, Y. Makino ${ }^{5}$, K. Masuda ${ }^{5}$, E. Matsubayashi ${ }^{5}$, H. Menjo ${ }^{8}$, G. Mitsuka ${ }^{2,5}$, Y. Muraki ${ }^{5}$, Y. Okuno ${ }^{5,9}$, P. Papini ${ }^{1}$, A-L. Perrot ${ }^{10}$, S. Ricciarini $^{1,2}$, T. Sako ${ }^{5}$, N. Sakurai ${ }^{6}$, Y. Sugiura ${ }^{5}$, T. Suzuki ${ }^{7}$, T. Tamura ${ }^{11}$, A. Tiberio ${ }^{1,2}$, S. Torii ${ }^{6}$, A. Tricomi $^{12,13}$, W.C. Turner ${ }^{14}$, and Q.D. Zhou ${ }^{5}$

${ }^{1}$ INFN section of Florence, Italy

${ }^{2}$ University of Florence, Italy

${ }^{3}$ IFAC-CNR, Italy

${ }^{4}$ Ecole-Polytechnique, France

${ }^{5}$ Solar-Terrestrial Environment Laboratory, Nagoya University, Japan

${ }^{6}$ Kobayashi-Maskawa Institute for the Origin of Particles and the Universe, Nagoya University,Japan

${ }^{7}$ RISE, Waseda University, Japan

${ }^{8}$ Graduate school of Science, Nagoya University, Japan

${ }^{9}$ Nagasaki Institute Applied Science, Japan

${ }^{10}$ CERN, Switzerland

${ }^{11}$ Kanagawa University, Japan

${ }^{12}$ INFN Section of Catania, Italy

${ }^{13}$ University of Catania, Italy

${ }^{14}$ LBNL, Berkeley, USA

\begin{abstract}
The LHCf experiment, optimized for the study of forward physics at LHC, completes its main physics program in this year 2015 , with the proton-proton collisions at the energy of $13 \mathrm{TeV}$. LHCf gives important results on the study of neutral particles at extreme pseudo-rapidity, both for proton-proton and for proton-ion interactions. These results are an important reference for tuning the models of the hadronic interaction currently used for the simulation of the atmospheric showers induced by very high energy cosmic rays. The results of this analysis and the future perspective are presented in this paper.
\end{abstract}

\section{Introduction}

LHC-forward experiment (LHCf) measures neutral particles production in a very forward region in proton-proton and proton-ion collisions at the Large Hadron Collider. The main purpose of LHCf is to improve hadronic interaction models used by Monte Carlo (MC) simulations of cosmic ray experiments. Highest energy cosmic rays can only be detected from secondary particles which are produced by the interaction of the primary particles with nuclei of the atmosphere, the so-called air showers. It is possible to reconstruct the kinematic parameters and the type of primary particle

a e-mail: marina.delprete@fi.infn.it 
studying the development of air showers. The energy flow of secondary particles is concentrated in the forward direction. Measurement of particle production at lager pseudo-rapidity, small angles, are very important. Moreover in the forward region soft QCD interactions dominates this MC simulations of air showers are based on phenomenological model, therefore inputs from experimental data are crucial. LHC accelerator gives the possibility to study a wide range of collision energies, from 0.9 $\mathrm{TeV}$ to $13 \mathrm{TeV}$ in the center of mass frame, which corresponds to an energy range in the laboratory frame from $10^{14} \mathrm{eV}$ to $10^{17} \mathrm{eV}$, this energy range covers the "knee" region of cosmic rays spectrum, which occurs at $10^{15} \mathrm{eV}$.

For these reasons LHCf can give more information to improve hadronic interaction models used in $\mathrm{MC}$ simulations to reproduce the development of air showers.

\section{The LHCf experiment}

LHCf is composed of two independent detectors, called Arm1 and Arm2 located 140 meters away from ATLAS interaction point, IP1 [1]. Arm1 is placed in the side of IP8 direction, toward LHCb experiment, and Arm2 is in the opposite direction toward IP2 and ALICE experiment. Detectors are placed inside Target Neutral Absorber (TAN), where the beam pipe turns into two separates tubes. The LHCf detectors occupy the first $30 \mathrm{~cm}$ of the TAN slots, facing the interaction point, thus minimizing the material between them and IP1. This is the best condition allowed at the LHC for measuring fluxes and energy spectra of high energy forward neutral particles produced by the interacting beams. Since charged particles are deviated by the D1 dipole magnet (which bends colliding beams into the two separate beam pipes), only neutral particles, mainly photons and neutrons, reach the detector.

Each detector is made of two sampling and imaging calorimeters called towers. This is required to minimize the overlap of signals due to the different simultaneous showers when detecting two gamma system decay. The longitudinal dimension of towers is of 44 radiation lengths, which correspond to 1.6 nuclear interaction lengths. The transverse cross sections are different for the two detectors. Arm1 has dimension $20 \times 20 \mathrm{~mm}^{2}$ for the small tower and for the large tower $40 \times 40 \mathrm{~mm}^{2}$. The Arm2 transverse cross sections are $25 \times 25 \mathrm{~mm}^{2}$ for the small tower and $32 \times 32 \mathrm{~mm}^{2}$ for the large tower.

Each tower is composed of 16 tungsten layers and 16 plastic scintillator layers to measure energy and also contains 4 position sensitive layers that are different for the two detector [2]. Arm1 uses scintillating fiber ( $\mathrm{SciFi}$ ) to measure position of the neutral particles in the towers, in Arm2 the position is measured by silicon microstrip detectors.

The different geometries of the two detectors have been carefully studied to maximize the accessible kinematic region and exploit the different characteristics of the position sensitive detectors. The smaller tower of each detector is placed on the beam center and covers the pseudo-rapidity range $\eta>$ 9.6, while the larger tower covers the pseudo-rapidity range $8.4<\eta<9.4$. Energy resolution obtained with these detectors configuration is better than $5 \%$ for photons and of about $40 \%$ for neutrons.

\section{Results from LHCf}

Until now, data were taken in three different runs of LHC in 2010, 2013 and 2015. In 2010 LHC produced proton-proton collisions at $\sqrt{s}=900 \mathrm{GeV}$ and at $\sqrt{s}=7 \mathrm{TeV}$; in 2013 proton-lead collisions at $\sqrt{s_{N N}}=5.02 \mathrm{TeV}$ and proton-proton collisions at $\sqrt{s}=2.76 \mathrm{TeV}$ were produced. In 2015 a special low luminosity proton-proton run at $\sqrt{s}=13 \mathrm{TeV}$ was dedicated to LHCf. Main results by the LHCf experiment are published in papers [3-6]. In this section we will present results for the inclusive neutron spectra measured in p-p collisions at $\sqrt{s}=7 \mathrm{TeV}$ in section 3.1, for the $p_{T}$ vs the pseudo rapidity $\pi_{0}$ spectra in $\mathrm{p}-\mathrm{Pb}$ collisions at $\sqrt{s_{N N}}=5.02 \mathrm{TeV}$ in section 3.2 and preliminary analysis from the data taken in proton-proton collisions at $\sqrt{s}=13 \mathrm{TeV}$ in section 3.3. 


\subsection{Neutron spectra in p-p collisions at $\sqrt{s}=7 \mathrm{TeV}$}

The data used in this analysis were taken in 2010 in low luminosity conditions, that are optimal for LHCf experiment due to the reduced event pile-up effect. Integrated luminosities were $0.68 n b^{-1}$ and $0.53 \mathrm{nb}^{-1}$ for Arm1 and Arm2 respectively, with a typical luminosity of $6.4 \cdot 10^{28} \mathrm{~cm}^{-2} \mathrm{~s}^{-1}$, derived from LHCf front counters [7]. Performance of detectors for hadron measurements have been carefully studied by Monte Carlo simulations. The data analysis method for neutron energy spectra closely follows that used for the photon component, which is described in [8].
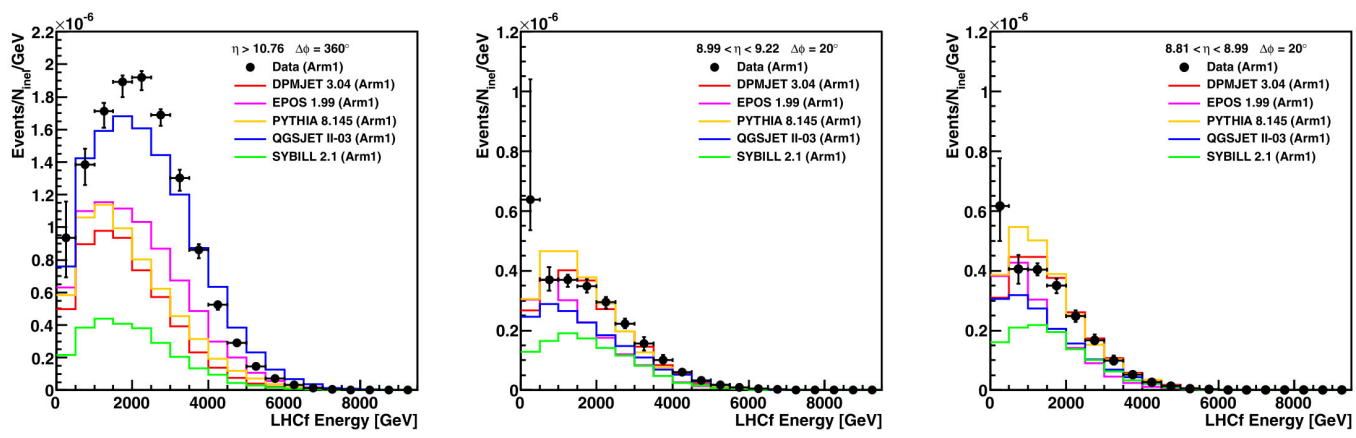

Figure 1. Preliminary neutron spectra measured by LHCf Arm1 in the $\sqrt{s}=7 \mathrm{TeV}$ p-p collisions in three different rapidity regions. $\eta$ is $>10.75,8.99<\eta<9.22$ and $8.81<\eta<8.99$, in the left, central and right plots respectively.

Particle identification has been carried out by looking at the longitudinal shower development, using two dimensional cuts in the L20\% and L90\% plane, where $L 20 \%$ and $L 90 \%$ are the longitudinal depths containing $20 \%$ and $90 \%$ of the total deposited energy, respectively. A two dimensional cut on parameter $L 2 D=L 90 \%-1 / 4 \cdot L 20 \%$ is then applied. Impact position of particle is reconstructed by the transverse distribution of energy deposit in position sensitive layer. Since the energy resolution
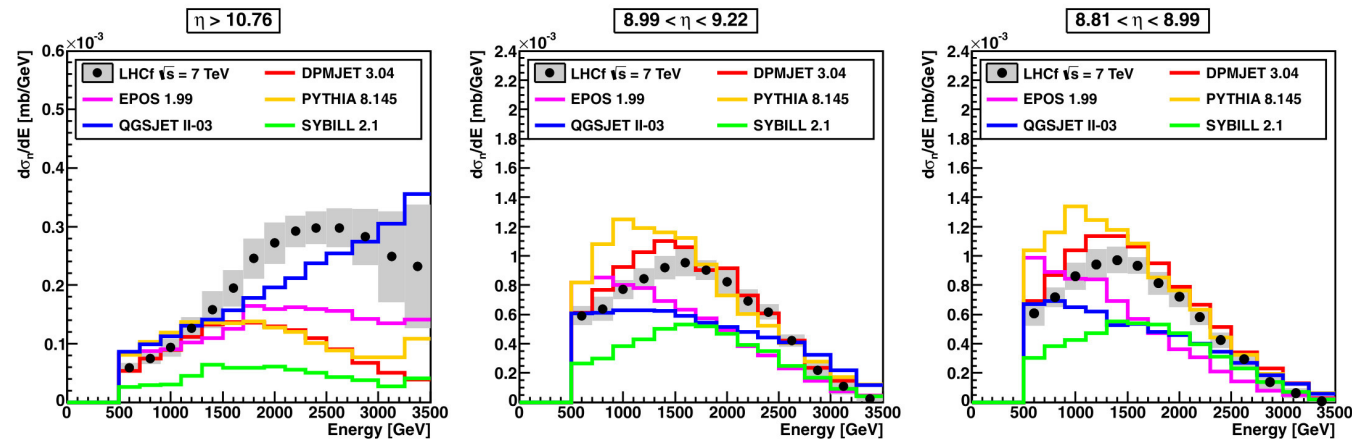

Figure 2. Preliminary neutron spectra measured by LHCf in the $\sqrt{s}=7 \mathrm{TeV}$ p-p collisions in three different rapidity regions, compared with the models predictions.

for neutrons is of about $40 \%$, true energy spectra are smeared by detector response and it is necessary 
to apply leakage corrections to the energy. Smeared energy spectra of neutrons measured by Arm1 and Arm 2 are compared with MC simulation predictions of several models are shown in figure 1 in three pseudo-rapidity ranges. Unfolding technique is therefore needed to estimate true energy spectra, we use a multidimensional-spectra unfolding Bayesian method relying on energy and $P_{T}$ measured variables [9]. Preliminary results after unfolding are shown in figure 2. A pronounced high energy neutron peak in the very forward region $(\eta>10.76)$, that is predicted only by the QGSJET-II model, is evident from these results. The presence of this peak is a clear indication of small inelasticity in the very forward direction of the p-p collision. We therefore expect that the baryons produced in the first interaction of a primary cosmic ray with the atmosphere carry on a large fraction of the primary energy, leading to deeply penetrating VHECR induced atmospheric showers.

\section{$3.2 \pi_{0}$ spectra in p-Pb collision at $\sqrt{s_{N N}}=5.02 \mathrm{TeV}$}

During $2013 \mathrm{p}-\mathrm{Pb}$ run at the nucleon-nucleon center-of-mass energy of $\sqrt{s_{N N}}=5.02 \mathrm{TeV}$, Arm2 was installed on p-remnant side for almost all the time, but it also operated for some hours on the $\mathrm{Pb}$ remnant side. The spectra measured in the p-remnant side provide new information on the effect of

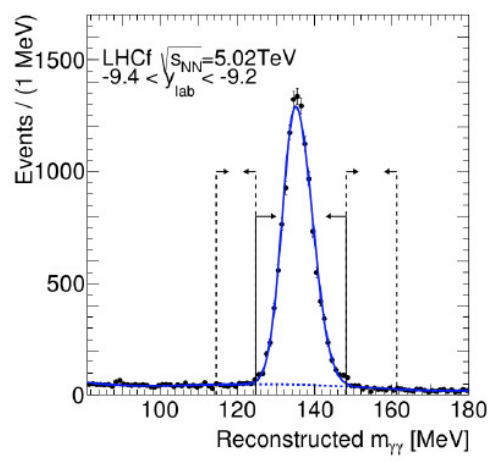

Figure 3. Two-photon invariant mass distributions in rapidity range $-9.4<y_{L A B}<-9.2$. Peak is fitted with an asymmetric Gaussian (blue solid curve), background is fitted with a polynomial (blue dashed curve). Signal and background windows are indicated by solid and dashed black lines respectively.

the dense nuclear matter in the forward particle production, which is particularly important for HECR Physics. Infact heavy nuclei and light nuclei can be involved in the the primary interactions of cosmic rays in the Earth atmosphere [10].

The invariant mass spectrum of the photons pairs for events where each calorimeter tower is hit by one photon, in figure 3, demonstrates the good performance of the detector in the reconstruction of a clean $\pi_{0}$ sample. A clean peak corresponding to the neutral pion events is prominent over a small background due to pairs of uncorrelated photons. Once the $\pi_{0}$ events are identified, transverse momentum and rapidity are evaluated, neutral pion $P_{T}$ spectra is measured in different rapidity ranges, and the results have been compared to the predictions of different hadronic interaction models. According to preliminary simulations, approximately half of the forward produced $\pi_{0}$ comes from UltraPeripheral Collisions (UPC) of the proton in the electromagnetic field produced by the $\mathrm{Pb}$ nucleus. To obtain pure QCD spectra the effect of the UPC is subtracted. The Weizsacher-Williams approximation is used to estimate the energy distribution of the virtual photons emitted by the fully ionized $\mathrm{Pb}$ nucleus, 

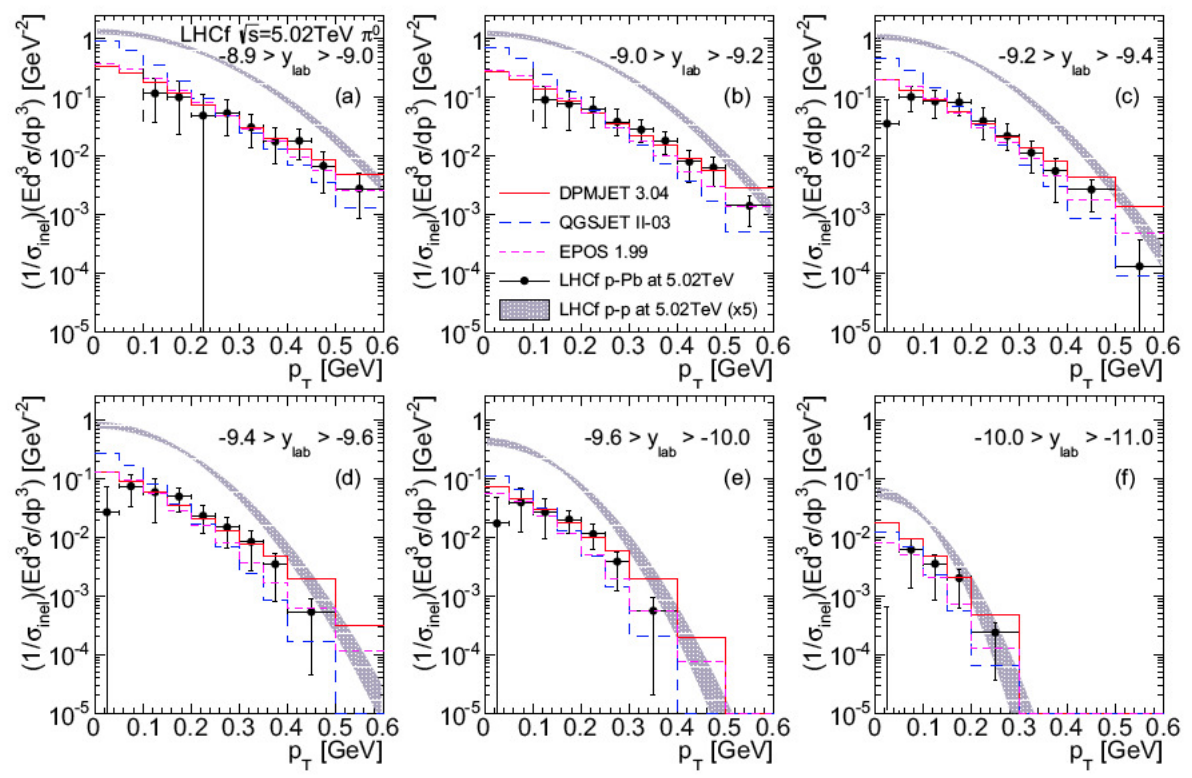

Figure 4. Transverse momentum distribution of neutral pions in several rapidity regions. Coloured histograms show results from Monte Carlo simulations with different hadronic interaction models, black points show experimental data in $\mathrm{p}-\mathrm{Pb}$ collisions at $5.02 \mathrm{TeV}$, gray shaded areas represent the extrapolation at $5.02 \mathrm{TeV}$ from data with p-p collisions (multiplied by a factor 5 ).

and finally the electromagnetic interactions between the virtual photons and the proton is simulated with the SOPHIA model [11].

Neutral pion $P_{T}$ spectra were measured in different rapidity ranges, and the results were compared to the predictions of different hadronic interaction MC models. Moreover spectra are compared with the spectra in $\mathrm{p}-\mathrm{p}$ collisions at $5.02 \mathrm{TeV}$. Th p-p spectra were estimated by interpolating the LHCf results from the p-p runs at $2.76 \mathrm{TeV}$ and $7 \mathrm{TeV}$ taken in the 2013 and 2010 respectively. In figure 4 are shown the results. The better data agreement is with the EPOS and DPMJET simulations and the $P_{T}$ spectra in $\mathrm{p}-\mathrm{Pb}$ collisions are harder than in $\mathrm{p}-\mathrm{p}$ collisions.

Figure 5 shows the measured $R_{p P b}$ compared with the predictions by different models. LHCf data show a strong suppression for neutral pion production for rapidity values $y_{l a b}>8.9 . R_{p P b}$ varies from 0.1 at $P_{T} \sim 0.1 \mathrm{GeV}$ to 0.3 at $P_{T} \sim 0.6 \mathrm{GeV}$. Hadronic interaction models predict also small values of $R_{p P b}$, showing an overall agreement with the LHCf data within the experimental uncertainty

\subsection{Preliminary analysis at $\sqrt{s}=13 \mathrm{TeV} \mathbf{p}-\mathbf{p}$ run}

In June 2015 there was a special low luminosity p-p run at $\sqrt{s}=13 \mathrm{TeV}$ was dedicated for LHCf data measurement. The luminosity was $10^{29} \mathrm{~cm}^{-2} \mathrm{~s}^{-1}, \sim 5$ orders of magnitudes below the nominal one of LHC. In preparation of this data taken both the detectors were upgraded. Due to the higher radiation damage expected, all plastic scintillators were replaced by GSO scintillators, with larger radiation hardness. Also scintillating fiber in Arm1 were replaced by GSO bars and in Arm2 silicon detectors position were changed to better catch showers in calorimeters and a new microstrip bonding scheme 

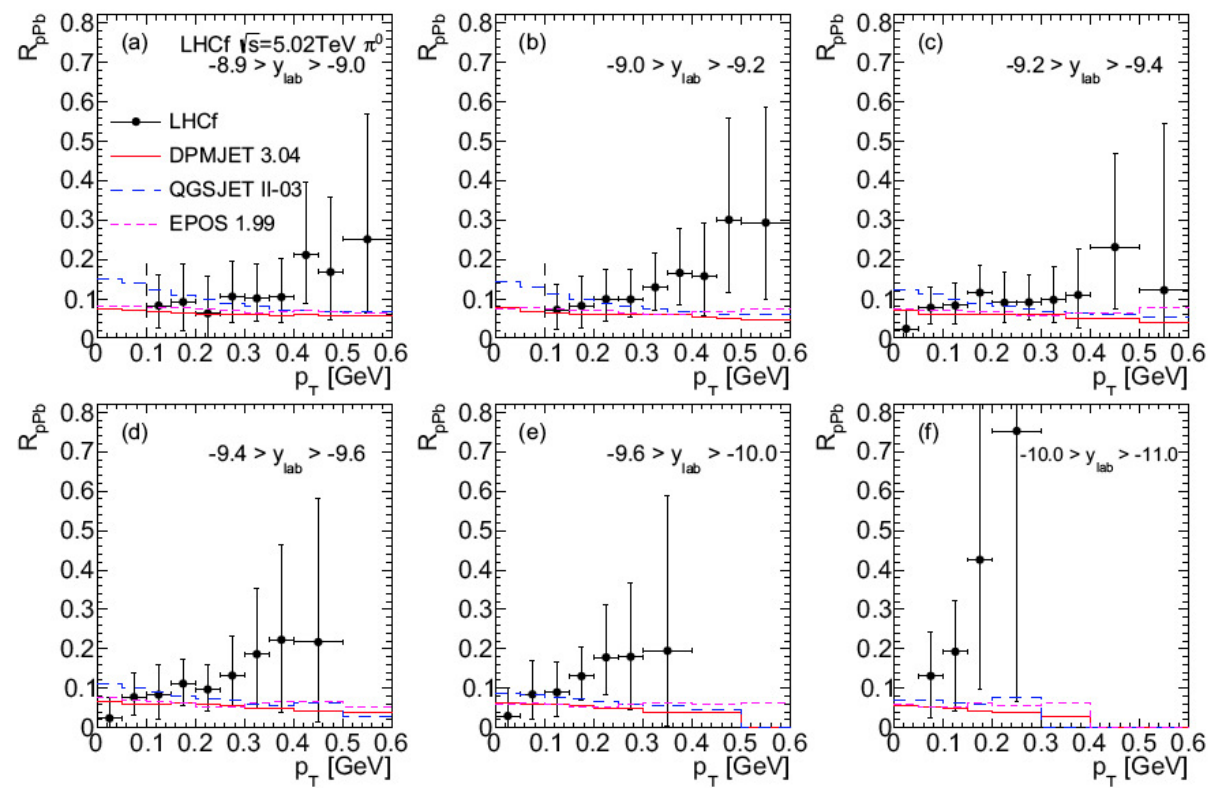

Figure 5. Nuclear modification factor for neutral pions in $\mathrm{p}-\mathrm{Pb}$ collisions at $5.02 \mathrm{TeV}$. Coloured histograms show results from Monte Carlo simulations with different hadronic interaction models, experimental data are shown as black points. Nuclear modification factor grows with energy as expected.

has been done to reduce the signal amplitude and avoid saturation of electronics with higher energy signals [12]. From $8^{\text {th }}$ to $12^{\text {nd }}$ of June LHCf takes successfully more than 32 hours of physics data . This operation was in collaboration with the ATLAS experiment, the trigger signals of LHCf were sent to ATLAS that recorded their data accordingly. The analyses of common events will permit to classify the events based on the nature of the processes, diffractive dissociation and non-diffractive. Selecting only diffractive events using ATLAS informations will improve the study of soft QCD interactions.

The high statistic and the good performance of detectors have permitted to detect clearly during the operation, with a preliminary analysis, the mesons $\pi_{0}$ and $\eta$, in the invariant mass of the two gammas (figure 6).

\section{Conclusion and future prospects}

LHCf experiment has successfully completed the first proposed program. LHCf performed measurements on forward production of neutral particles in proton-proton and proton-lead collisions at LHC accelerator. These measurements are necessary to calibrate hadronic interaction models used in cosmic rays physics to understand the development of atmospheric showers. We measured photons, neutral pions and neutrons for p-p interactions at $\sqrt{s}=900 \mathrm{GeV}$ and $2.76,7,13 \mathrm{TeV}$ and $\mathrm{p}-\mathrm{Pb}$ interactions at $\sqrt{s}=5.02 \mathrm{TeV}$.

The analysis of hadronic component for p-p $7 \mathrm{TeV}$ run and the $\pi_{0}$ analysis for $\mathrm{p}-\mathrm{Pb}$ run are completed. The analysis of data taken in p-p run at $13 \mathrm{TeV}$ is ongoing as the the analysis in common with Atlas collaboration on data taking in 2013 is ongoing. 


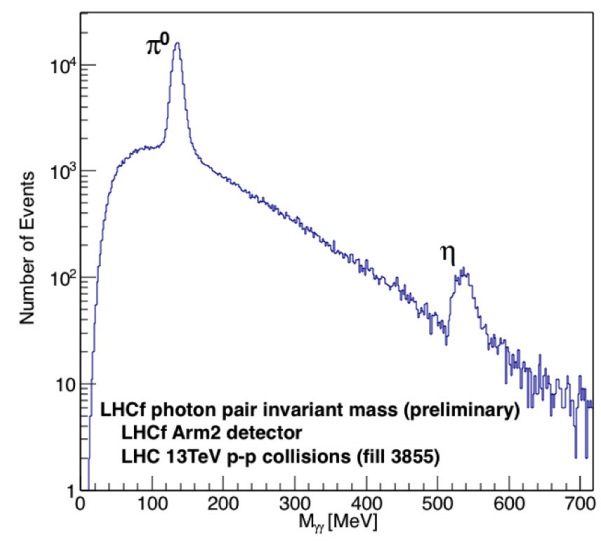

Figure 6. Photon pair invariant mass obtained with the data of the fill 3855 in Arm2. The mass of $\pi_{0}$ and $\eta$ are clearly reconstructed at $\sim 140 \mathrm{MeV}$ and $\sim 550 \mathrm{MeV}$ respectively.

The future prospects of the experiment are to complete the data taking with a $\mathrm{p}-\mathrm{Pb}$ run at the top LHC energy in the 2016 or 2017 . Another opportunity will given moving Arm1 detector to RICH, and LHCf will be RICHf, and take data in p-p collisions at $510 \mathrm{TeV}$ in the 2017.

\section{References}

[1] O. Adriani et al., JINST 3, S08006 (2008)

[2] O. Adriani et al., JINST 5, P01012 (2010)

[3] O. Adriani et al., Physics Letters B 715, 298-303 (2012)

[4] O. Adriani et al., Physics Letters B 703, 128-134 (2011)

[5] O. Adriani et al., Physical Review D 86, 092001 (2012)

[6] O. Adriani et al., Physical Review C 89, 065209 (2014)

[7] K. Taki et al., JINST 7, T01003 (2012)

[8] K. Kawade et al., JINST 9, P03016 (2014)

[9] G. D' Agostini, Nucl. Instrum. Meth. A 362, 487 (1995)

[10] O. Adriani, et al., CERN-LHCC-2011-015, LHCC-I-021 (2011)

[11] A. Mucke et al., Computer Physics Communications 124, 290 (1999)

[12] Y. Makino et al. PoS TIPP2014 028, (2014) 